\title{
First-line palliative chemotherapy clinical benefit is a key determinant of survival in metastatic uterine leiomyosarcoma
}

\author{
๑Dmrah Eraslan, (D)Aş̧egül İlhan, ĐFatih Yıldız, @Mutlu Doğan \\ University of Health Sciences, Dr. Abdurrahman Yurtaslan Ankara Oncology Training and Research Hospital, Department of Medical Oncology, \\ Ankara, Turkey
}

Cite this article as: Eraslan E, İlhan A, Ylldız F, Doğan M. First-line palliative chemotherapy clinical benefit is a key determinant of survival in metastatic uterine leiomyosarcoma. J Health Sci Med 2021; 4(6): 795-799.

\begin{abstract}
Aim: To evaluate whether obtaining a clinical benefit with first-line therapy in patients receiving palliative systemic therapy with a diagnosis of metastatic uterine leiomyosarcoma (ULMS) provides a survival benefit and the factors that may predict first-line therapy response.

Material and Method: This study was a retrospective observational single-center analysis conducted with patients diagnosed with metastatic ULMS. Patients who received palliative chemotherapy with an ECOG PS of 0 or 1 at the time of diagnosis of metastatic disease were included in the study. Main patient characteristics, first-line palliative treatment responses, progressionfree survival, and overall survival (OS) were reviewed retrospectively. Multivariate analyses were performed to determine the independent predictive factors of first-line palliative treatment response and overall survival.

Results: Of the 36 patients whose medical records were evaluated retrospectively, 24 patients who were eligible for the study were included in the study. Gemcitabine plus docetaxel combination chemotherapy was the most commonly used treatment protocol $(\mathrm{n}=12,50 \%)$ for first-line palliative treatment. While a complete response as a first-line treatment response could not be achieved, clinical benefit (partial remission and stable disease) and progressive disease were observed in 15 (62\%) and 9 (37.5\%) patients, respectively. Binary logistic regression analysis failed to detect any independent predictive factors for the clinical benefit of first-line palliative therapy. Median (OS) was 19.7 (95\% CI, 4.1-35.3) months for all patients (N=24). Median OS was 25.6 (95\% CI, 21.0-30.2) months and 6.9 (95\% CI, 1.7-12.2) months for patients with and without the clinical benefit of first-line palliative chemotherapy $(\mathrm{p}=0.004)$. Cox-regression analysis revealed that increasing age at diagnosis of metastatic disease $(\mathrm{HR}=0.929,95 \% \mathrm{CI} 0.870-0.992$, $\mathrm{p}=0.027)$, pulmonary metastasectomy $(\mathrm{HR}=0.162,95 \% \mathrm{CI} 0.031-0.863, \mathrm{p}=0.033)$, and presence of first-line palliative chemotherapy clinical benefit $(\mathrm{HR}=0.195,95 \%$ CI $0.063-0.606, \mathrm{p}=0.005)$ were independent predictive factors for a better OS.

Conclusion: In metastatic ULMS, for which the survival benefit is not clear with palliative chemotherapy, prolonged survival can be obtained in patients with clinical benefit with first-line palliative chemotherapy. There is a need for new studies to determine the factors that will predict the clinical benefit of first-line palliative chemotherapy.
\end{abstract}

Keywords: Metastatic uterine leiomyosarcoma, palliative chemotherapy, clinical benefit, overall survival

\section{INTRODUCTION}

Uterine sarcomas are highly aggressive mesenchymal tumors and make up less than five percent of all malignant uterine tumors (1). Although there are numerous histologic subtypes, leiomyosarcomas are the most common (nearly $70 \%)$ subgroup of uterine sarcomas $(2,3)$. The main goal in treating early-stage disease is complete surgical resection of the tumor bulk (4). The clinical course of uterine leiomyosarcomas (ULMSs) is quite aggressive; even with successful surgical treatment, there is a chance of recurrence of up to 70\% (5). Approximately twenty percent of patients are stage IV at diagnosis (6). In the presence of isolated metastases, a metastasectomy accompanying systemic palliative treatments with and without radiotherapy may be an appropriate treatment approach (7). Also, systemic therapy should be considered in patients who are not suitable candidates for surgery because of poor performance status or high tumor burden. Although these are among the treatment options, it is suggested that leiomyosarcomas are not sensitive to radiotherapy and chemotherapy (8). In a study in which 5 -year disease-specific survival was reported as $28.7 \%$ for stage IV ULMS, chemotherapy and radiotherapy could 
not have been shown to provide a survival benefit (6). Although there is no agreed-upon chemotherapy regimen for the treatment of advanced ULMS, the addition of docetaxel to gemcitabine monotherapy resulted in both better objective treatment response and improved survival for soft tissue sarcomas (9).

In our study, we aimed to evaluate whether obtaining a clinical benefit with first-line therapy in patients receiving palliative systemic therapy with the diagnosis of metastatic ULMS provides a survival benefit and the factors that may predict first-line therapy response.

\section{MATERIAL AND METHOD}

This study was a retrospective observational singlecenter analysis conducted with patients with a diagnosis of metastatic ULMS.

Ethical approval was obtained University of Health Sciences Dr. Abdurrahman Yurtaslan Ankara Oncology Training and Research Hospital Clinical Researchs Ethics Committee (Date: 13.01.2021, Decision No: 2021-01/942). All procedures were carried out in accordance with the ethical rules and the principles of the Declaration of Helsinki.

Patients who received palliative chemotherapy in the medical oncology clinic of the University of Health Sciences Dr. Dr. Abdurrahman Yurtaslan Ankara Oncology Training and Research Hospital between December 2010 and February 2021 were included. Medical records in the hospital data processing system and patient files were reviewed retrospectively. Patients older than 18 years of age and only those with an ECOG PS of 0 or 1 at the time of diagnosis of metastatic disease were included. Patients with an ECOG PS of 2 or more were excluded from the study. Patients' demographic characteristics, stages at the time of diagnosis, adjuvant treatment modalities, recurrence time, metastatic sites, whether metastasis surgery was performed, systemic palliative treatments, systemic treatment response rates were recorded. Time to metastasis was estimated as the time from surgery to the development of metastasis for patients who underwent curative surgery and zeroed for metastatic patients at diagnosis. Patients who achieved clinical benefit (complete response, partial remission, stable disease) or progressive disease response with first-line therapy were identified. Factors that may affect the clinical benefit of first-line therapy were evaluated. For first-line palliative systemic therapy, progression-free survival was defined as the time from initiation of therapy to progression. Overall survival (OS) was defined as the time from diagnosis of metastatic disease to death or last follow-up. Overall survival analysis was performed of patients with and without clinical benefit from first-line palliative therapy. Prognostic factors that could predict OS were analyzed. Study results were compared with the literature data.

\section{Statistical Analysis}

Descriptive statistics were used to show the distribution of the main characteristics of the population. A binary logistic regression model was constructed that includes factors that could predict the clinical benefit of firstline palliative systemic therapy. Survival rates were estimated using the Kaplan-Meier method, and groups were compared using the log-rank test for difference in survival. A Cox regression model was carried out that includes crucial factors that could predict death. Statistical analysis was performed using SPSS software (SPSS for Windows, version 24.0, SPSS Inc., Chicago, USA). All statistical tests were two-sided, and a $\mathrm{P}<0.05$ value was considered statistically significant.

\section{RESULTS}

Medical records of 36 patients with metastatic ULMS were reviewed retrospectively. Eight patients with an ECOG PS of 2 or higher and four patients with insufficient medical records were excluded. Twentyfour patients with a median age of $54.9(35.8-70.7)$ were included in our study. The median follow-up time was 13.3 (range, 2.3-58.8) months. Four (16.7\%) patients were in the metastatic stage, and $20(83.3 \%)$ patients underwent curative primary surgical treatment with an early-stage disease at the initial diagnosis. Median time to the metastatic stage was 17.1 (range, 0.0-123.6) months. The most common site of metastasis $(n=20,83.3 \%)$ was lung, and $5(20.8 \%)$ patients underwent pulmonary metastasectomy. Main patient and disease characteristics are shown in Table 1.

\begin{tabular}{|lcc|}
\hline Table 1. Main patient and disease characteristics & \\
\hline Parameter & $\begin{array}{c}\text { Number } \\
(\mathbf{N = 2 4 )}\end{array}$ & Percent (\%) \\
\hline Age, median (range) & 54.9 & $35.8-70.7$ \\
Menopausal status & & \\
$\quad$ Premenopausal & 10 & 41.7 \\
$\quad$ Postmenopausal & 14 & 58.3 \\
Stage at first diagnosis & & \\
$\quad$ Stage I & 14 & 58.3 \\
$\quad$ Stage II & 1 & 4.2 \\
$\quad$ Stage III & 4 & 16.7 \\
$\quad$ Stage IV & 5 & 20.8 \\
Presence of metastases at diagnosis & 4 & 16.7 \\
Primary surgery & 20 & 83.3 \\
Adjuvant chemotherapy & 10 & 41.7 \\
Adjuvant radiotherapy & 2 & 8.3 \\
Metastasis sites & & \\
$\quad$ Lung & 20 & 83.3 \\
$\quad$ Peritoneum & 7 & 29.2 \\
$\quad$ Bone & 4 & 16.7 \\
$\quad$ Liver & 4 & 16.7 \\
$\quad$ Lymph node & 2 & 8.3 \\
$\quad$ Only Lung & 10 & 41.7 \\
$\quad$ Lung with other sites & 10 & 41.7 \\
Only extrapulmonary & 4 & 16.7 \\
Metastasectomy & 5 & 20.8 \\
\hline
\end{tabular}


Twenty-four patients included in the study were given various palliative chemotherapy regimens. In addition, patients had received one to four lines of palliative systemic chemotherapy. Gemcitabine plus docetaxel combined chemotherapy was given most frequently $(n=20,50.0 \%)$ as first-line treatment. While clinical benefit was obtained with first-line chemotherapy in 15 (62.5\%) patients, the first-line treatment response of 9 (37.5\%) patients was progressive disease. Median progressionfree survival with first-line chemotherapy was 6.1 (95\% CI, 1.5-10.7) months. First-line chemotherapy regimens, the responses obtained with first-line treatment, and the number of patients receiving further treatments are shown in Table 2.

Median OS was 19.7 (95\% CI, 4.1-35.3) months for all patients $(\mathrm{N}=24)$ (Figure 1A). The OS rate at 36 months was $22.3 \%$ for all patients. Median OS was 25.6 (95\% CI, 21.0-30.2) months and 6.9 (95\% CI, 1.7-12.2) months for patients with and without the clinical benefit of firstline palliative chemotherapy, respectively $(\mathrm{p}=0.004)$ (Figure 1B). The OS rate at 36 months was $28.3 \%$ and $11.1 \%$ for patients with and without the clinical benefit

\begin{tabular}{|lcc|}
\hline Table 2. Features associated with palliative chemotherapies \\
\hline Parameter & $\begin{array}{c}\text { Number } \\
\text { (N=24) }\end{array}$ & $\begin{array}{c}\text { Percent } \\
\text { (\%) }\end{array}$ \\
\hline First- line chemotherapy & & \\
$\quad$ Gemcitabine plus docetaxel & 12 & 50.0 \\
$\quad$ IMA & 8 & 33.3 \\
$\quad$ Others & 4 & 16.7 \\
Best response with first-line chemotherapy & 4 & \\
$\quad$ Partial remission & 16.7 \\
$\quad$ Stable disease & 11 & 45.8 \\
$\quad$ Progressive disease & 9 & 37.5 \\
$\quad$ Clinic benefit rate (CR + PR + SD) & 15 & 62.5 \\
Patients receiving second-line therapy & 13 & 54.2 \\
$\begin{array}{l}\text { Patients receiving third-line therapy } \\
\text { Patients receiving fourth-line therapy }\end{array}$ & 9 & 37.5 \\
\hline $\begin{array}{l}\text { IMA, chemotherapy scheme consisting of ifosfamide, mesna and adriamycin; CR, } \\
\text { complete response; PR, partial remission; SD, stable disease }\end{array}$ & 3 \\
\hline
\end{tabular}

Table 3. Cox-regression analysis results including factors that may predict overall survival

\begin{tabular}{|c|c|c|c|c|}
\hline \multirow{2}{*}{ Parameter } & \multirow{2}{*}{ HR } & \multicolumn{2}{|c|}{ 95\% CI } & \multirow{2}{*}{ p-valu } \\
\hline & & Lower & Upper & \\
\hline Age & 0.929 & 0.870 & 0.992 & 0.027 \\
\hline $\begin{array}{l}\text { Presence of metastases at } \\
\text { diagnosis (Yes vs No) }\end{array}$ & 2.706 & 0.525 & 13.952 & 0.234 \\
\hline Time to metastasis & 1.005 & 0.980 & 1.030 & 0.726 \\
\hline $\begin{array}{l}\text { Only extrapulmonary } \\
\text { metastasis (Yes vs No) }\end{array}$ & 3.205 & 0.660 & 15.549 & 0.148 \\
\hline $\begin{array}{l}\text { Metastasectomy } \\
\text { (Yes vs No) }\end{array}$ & 0.162 & 0.031 & 0.863 & 0.033 \\
\hline $\begin{array}{l}\text { First-line CTx clinical } \\
\text { benefit (Yes vs No) }\end{array}$ & 0.195 & 0.063 & 0.606 & 0.005 \\
\hline CTx, chemotherapy & & & & \\
\hline
\end{tabular}

of first-line palliative chemotherapy, respectively. In the binary logistic regression analysis, in which factors (age, menopausal status, stage at diagnosis, primary surgical treatment, adjuvant chemotherapy, adjuvant radiotherapy, site of metastasis, time to metastasis) that may affect the benefit of first-line palliative chemotherapy were included, no independent predictive factor was found to predict the benefit of first-line palliative chemotherapy.

In Cox-regression analysis including factors that may predict OS, increasing age at diagnosis of metastatic disease $(\mathrm{HR}=0.929,95 \%$ CI $0.870-0.992, \mathrm{p}=0.027)$, pulmonary metastasectomy $(\mathrm{HR}=0.162,95 \% \mathrm{CI} 0.031$ $0.863, \mathrm{p}=0.033)$, and presence of first-line palliative chemotherapy clinical benefit $(\mathrm{HR}=0.195,95 \% \mathrm{CI}$ 0.063-0.606, $\mathrm{p}=0.005)$ were independent predictive factors for a better OS (Table 3 ).

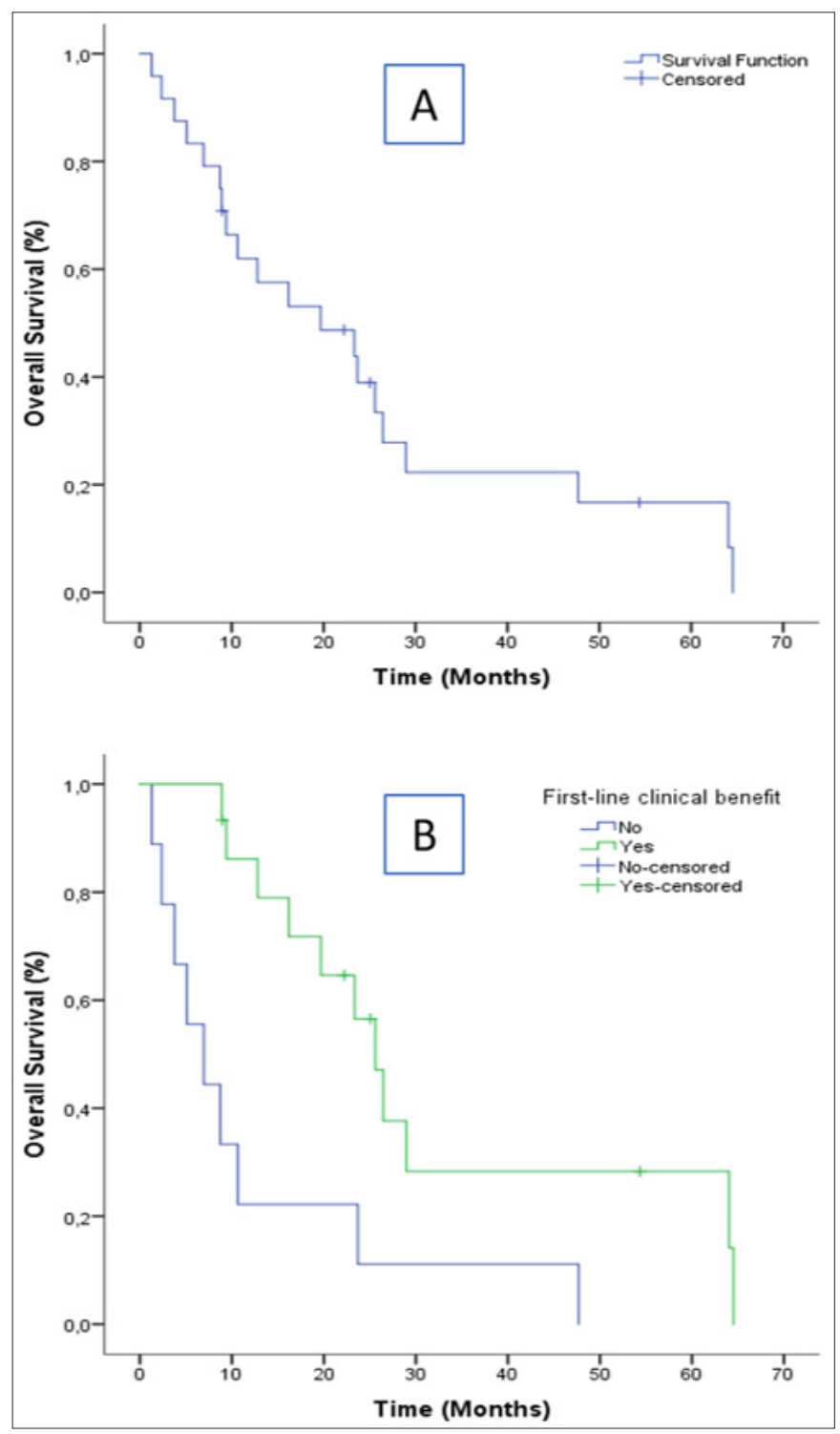

Figure 1. Kaplan-Meier curves of overall survival, A: Plot of all patients receiving palliative chemotherapy, B: Comparative plot of patients with and without the clinical benefit of first-line palliative chemotherapy. 


\section{DISCUSSION}

In our study, we observed that OS of patients who achieved clinical benefit with first-line palliative chemotherapy was better than those whose chemotherapy response was in the form of progressive disease. However, we were unable to demonstrate any independent predictive factors that could predict first-line therapy response. In multivariate analysis, we found that increasing age at diagnosis of metastatic disease, metastasectomy, and clinical benefit from first-line palliative chemotherapy were independent predictive factors for a better OS.

Leiomyosarcomas constitute the most common histological subtype of all soft tissue sarcomas (10). However, studies mainly cover all soft tissue sarcomas and include gastrointestinal stromal tumors that differ in their clinical course and treatment. The majority of studies evaluating the efficacy of chemotherapy in patients with advanced or metastatic uterine sarcoma concluded with negative results and no survival benefit (11). Moreover, since ULMS is a very rare disease, it is challenging to conduct a study in this patient group, and the literature data on chemotherapy efficacy for ULMS is limited. In a phase II study in which Hensley et al. (12) evaluated the efficacy of gemcitabine plus docetaxel treatment in 29 patients with metastatic ULMS, they achieved an objective response (complete and partial remission) in 53\% of the patients. Also, PFS and OS were reported at approximately six months and 18 months, respectively, in the same study (12). Although the treatment response rate in our study was considerably lower than that of Hensley et al., the PFS and OS in our study were almost the same as in this study. In addition, in another phase II study by Hensley et al. (13), an objective response rate of $35.8 \%$, approximately 4.5 months, and 16 months PFS and OS was obtained with fixed-dose rate gemcitabine plus docetaxel as first-line therapy for metastatic ULMS. The OS range in this study was quite wide, ranging from 4 to 41.3 months, and some patients lived very long with gemcitabine plus docetaxel therapy (13). Similarly, there was a fairly wide OS range in our study. In addition, there is a $17 \%$ difference between patients who are alive at the end of the third year compared to patients who have clinical benefit from first-line chemotherapy and those who do not. The literature data as mentioned above and our results suggest that chemotherapy may be beneficial for OS in some patients with metastatic ULMS. To the best of our knowledge, no prospective randomized study in which a significant OS benefit with chemotherapy for metastatic ULMS can be clearly demonstrated. However, in an observational cohort study of 7455 patients based on the 1998-2013 National Cancer Database by Seagle et al. (14), chemotherapy was associated with 8.5 (19.4 vs. $10.9, \mathrm{p}<0.001)$ months increased survival of women with metastatic leiomyosarcoma.
In a phase III study in which patients with a metastatic soft tissue sarcoma diagnosis showed a significant improvement of $12 \%$ in the overall response rate and nearly three months in PFS with the addition of ifosfamide to doxorubicin monotherapy, but no significant difference in OS (15). In this study, ULMS was not examined as a separate group, and factors that could predict death were not evaluated statistically. However, in the phase III GeDDiS study involving 257 patients with treatmentnaive metastatic soft tissue sarcoma, no difference was observed between patients treated with gemcitabine plus docetaxel and doxorubicin in terms of treatment response rates, PFS, and OS (16). Although a subgroup analysis was performed comparing leiomyosarcomas with other sarcoma subtypes and ULMS with other sarcoma subtypes, no subgroup analysis was performed in terms of factors that would predict treatment response (16). In order to minimize the effect of other factors on survival, we included patients with an ECOG PS of 0 or 1 at the start of treatment. Although we demonstrated in our study that providing disease control with firstline palliative chemotherapy was associated with more prolonged survival, we could not detect any factor that could predict treatment response. This result can be explained by the small number of patients and the retrospective nature of our study. However, there is a lack of literature data regarding the factors that can predict the palliative systemic treatment response in ULMS.

Previous studies have demonstrated that big tumor size, high mitotic index, high grade, advanced stage, advanced age, and inadequate surgical treatment are negative prognostic factors for ULMS $(5,6,17,18)$. However, none of these studies evaluated patients receiving palliative chemotherapy for the advanced disease alone. Our study differs from these studies in that the patient profile is a more specific group, and for this reason, we think that our study is valuable. In our study, increasing age at diagnosis of metastatic disease, metastasectomy, and clinical benefit from first-line palliative chemotherapy were positive prognostic factors. Bernstein-Molho et al. (19) observed a survival difference of approximately 14 months with metastasectomy in a study involving 33 patients with metastatic ULMS. In an analysis of 128 patients with recurrent ULMS, secondary cytoreductive surgery prolonged time to recurrence (20). Moreover, in the same analysis, neither chemotherapy nor radiation showed improved outcomes in patients with recurrent ULMS (20). Also, considering the high hazard ratios in our analysis, we can say that the presence of only extrapulmonary metastases and metastatic disease at the time of diagnosis may be negative prognostic factors. The inadequacy of the number of patients in our study may be the reason for not showing statistical significance for these factors. 


\section{CONCLUSION}

In metastatic ULMS, for which the survival benefit is not clear with palliative chemotherapy, prolonged survival can be obtained in patients with clinical benefit with first-line palliative chemotherapy. There is a need for new studies to determine the factors that will predict the clinical benefit of first-line palliative chemotherapy.

\section{ETHICAL DECLARATIONS}

Ethics Committee Approval: Approval for the study was given by the University of Health Sciences Dr. Abdurrahman Yurtaslan Ankara Oncology Training and Research Hospital Clinical Researchs Ethics Committee (Date: 13.01.2021, Decision No: 2021-01/942)

Informed Consent: Because the study was designed retrospectively, no written informed consent form was obtained from patients.

Referee Evaluation Process: Externally peer-reviewed. Conflict of Interest Statement: The authors have no conflicts of interest to declare.

Financial Disclosure: The authors declared that this study has received no financial support.

Author Contributions: All of the authors declare that they have all participated in the design, execution, and analysis of the paper, and that they have approved the final version.

\section{REFERENCES}

1. D’Angelo E, Prat J. Uterine sarcomas: a review. Gynecol Oncol 2010; 116: 131-9.

2. Society AC. Cancer facts \& figures 2018. Atlanta, GA2018.

3. Ricci S, Stone RL, Fader AN. Uterine leiomyosarcoma: Epidemiology, contemporary treatment strategies and the impact of uterine morcellation. Gynecol Oncol 2017; 145: 208-16.

4. Ganjoo KN. Uterine sarcomas. Curr Probl Cancer 2019; 43: 283-8.

5. Giuntoli RL, 2nd, Metzinger DS, DiMarco CS, et al. Retrospective review of 208 patients with leiomyosarcoma of the uterus: prognostic indicators, surgical management, and adjuvant therapy. Gynecol Oncol 2003; 89: 460-9.

6. Kapp DS, Shin JY, Chan JK. Prognostic factors and survival in 1396 patients with uterine leiomyosarcomas: emphasis on impact of lymphadenectomy and oophorectomy. Cancer. 2008; 112: 820-30.

7. Kanjeekal S, Chambers A, Fung MF, Verma S. Systemic therapy for advanced uterine sarcoma: a systematic review of the literature. Gynecol Oncol 2005; 97: 624-37.

8. Li J, Chen X, Hu X. High-intensity focused ultrasound for treatment of recurrent uterine leiomyosarcoma: a case report and literature review. J Int Med Res 2020; 48: 300060520942107.

9. Maki RG, Wathen JK, Patel SR, et al. Randomized phase II study of gemcitabine and docetaxel compared with gemcitabine alone in patients with metastatic soft tissue sarcomas: results of sarcoma alliance for research through collaboration study 002. J Clin Oncol 2007; 25: 2755-63.

10. Toro JR, Travis LB, Wu HJ, Zhu K, Fletcher CD, Devesa SS. Incidence patterns of soft tissue sarcomas, regardless of primary site, in the surveillance, epidemiology and end results program, 1978-2001: An analysis of 26,758 cases. Int J Cancer 2006; 119: 2922-30.
11. Desar IME, Ottevanger PB, Benson C, van der GraafWTA. Systemic treatment in adult uterine sarcomas. Crit Rev Oncol Hematol 2018; 122: $10-20$

12. Hensley ML, Maki R, Venkatraman E, et al. Gemcitabine and docetaxel in patients with unresectable leiomyosarcoma: results of a phase II trial. J Clin Oncol 2002; 20: 2824-31.

13. Hensley ML, Blessing JA, Mannel R, Rose PG. Fixed-dose rate gemcitabine plus docetaxel as first-line therapy for metastatic uterine leiomyosarcoma: a Gynecologic Oncology Group phase II trial. Gynecol Oncol 2008; 109: 329-34.

14. Seagle BL, Sobecki-Rausch J, Strohl AE, Shilpi A, Grace A, Shahabi S. Prognosis and treatment of uterine leiomyosarcoma: A National Cancer Database study. Gynecol Oncol 2017; 145: 61-70.

15. Judson I, Verweij J, Gelderblom H, et al. Doxorubicin alone versus intensified doxorubicin plus ifosfamide for first-line treatment of advanced or metastatic soft-tissue sarcoma: a randomised controlled phase 3 trial. Lancet Oncol 2014; 15: 415-23.

16. Seddon B, Strauss SJ, Whelan J, et al. Gemcitabine and docetaxel versus doxorubicin as first-line treatment in previously untreated advanced unresectable or metastatic soft-tissue sarcomas (GeDDiS): a randomised controlled phase 3 trial. Lancet Oncol 2017; 18: 1397-410.

17. Abeler VM, Røyne O, Thoresen S, Danielsen HE, Nesland JM, Kristensen GB. Uterine sarcomas in Norway. A histopathological and prognostic survey of a total population from 1970 to 2000 including 419 patients. Histopathology 2009; 54: 355-64.

18. Mayerhofer K, Obermair A, Windbichler G, et al. Leiomyosarcoma of the uterus: a clinicopathologic multicenter study of 71 cases. Gynecol Oncol 1999; 74: 196-201.

19. Bernstein-Molho R, Grisaro D, Soyfer V, Safra T, Merimsky O. Metastatic uterine leiomyosarcomas: a single-institution experience. Int J Gynecol Cancer 2010; 20: 255-60.

20. Giuntoli RL, 2nd, Garrett-Mayer E, Bristow RE, Gostout BS. Secondary cytoreduction in the management of recurrent uterine leiomyosarcoma. Gynecol Oncol 2007; 106: 82-8. 